\title{
Development of microsatellite loci for Taxus wallichiana var. wallichiana (Taxaceae) and cross-amplification in Taxaceae
}

\author{
B.B. Cheng*, Q.W. Sun* and Y.Q. Zheng \\ Beijing, China \\ *These authors contributed equally to this study. \\ Corresponding author: Y.Q. Zheng \\ E-mail: zyq8565@126.com
}

State Key Laboratory of Tree Genetics and Breeding, Key Laboratory of Silviculture of the State Forestry Administration, Lab of Molecular Identification of Plant Varieties, Research Institute of Forestry, Chinese Academy of Forestry, Haidian District,

Genet. Mol. Res. 14 (4): 16018-16023 (2015)

Received August 9, 2015

Accepted October 1, 2015

Published December 7, 2015

DOI http://dx.doi.org/10.4238/2015.December.7.15

ABSTRACT. Nine polymorphic microsatellite loci were isolated and characterized in Taxus wallichiana var. wallichiana, an endangered species in China. The number of alleles per locus ranged from 2 to 20 . Observed and expected heterozygosities varied from 0.0260 to 0.5325 and 0.3603 to 0.9231 , respectively. Positive cross-amplification of the 9 loci was observed in 2 other varieties of $T$. wallichiana and 4 other Taxaceae species. These loci will be of value for studying population genetic structures and for genetic resource conservation in T. wallichiana and other Taxus species.

Key words: Taxus wallichiana var. wallichiana; Microsatellite loci; Cross-amplification; Genetic structure 


\section{INTRODUCTION}

Taxus wallichiana var. wallichiana Zucc., an evergreen shrub or tree species native to Sichuan, Tibet and Yunnan in China, frequently occurs along streams at altitudes from 2000$3500 \mathrm{~m}$ (Li and Fu, 1997; Fu et al., 1999; Farjon, 2001). This species is scattered in broad-leaved and mixed forests, and is often associated with bamboos (Li and Fu, 1997; Fu et al., 1999). It is widely used for Taxol (paclitaxel) extraction and production of wood for high-quality furniture and ornamental purposes. These important uses have resulted in considerable interest in the species over the last few decades and have led to over-exploitation of wild resources through destructive harvesting, which has caused substantial loss of genetic diversity. T. wallichiana is currently listed as a rare and endangered species on China's national Red List (Xinqiang et al., 1996). Protection of the species requires that harvesting from natural forests is prohibited immediately and measures need to be taken to conserve genetic diversity.

To understand the genetic diversity within T. wallichiana, it will be necessary to develop an appropriate panel of genetic markers. Simple sequence repeat (SSR) markers offer a powerful tool for characterizing genetic diversity and have been widely used for genome mapping, population genetics, and related areas (Ellegren, 2004; Kalia et al., 2011; Zalapa et al., 2012). In recent years, several SSR markers have been developed for different species of the genus Taxus, such as T. sumatrana (Huang et al., 2008), T. yunnanensis (Miao et al., 2014), T. baccata (Dubreuil et al., 2008), T. chinensis var. mairei (Zhou et al., 2009), and T. wallichiana (Yang et al., 2009).

However, the previously developed SSR markers for T. wallichiana have identified few polymorphisms. As polymorphic SSR markers are needed to characterize genetic diversity and phylogeography of the species, we initiated this study to develop and characterize new SSR markers for T. wallichiana var. wallichiana, that could be also be applied across the whole of the Taxaceae family. Here, we describe the development of new SSR markers and apply them to two T. wallichiana varieties (T. wallichiana var. mairei and T. wallichiana var. chinensis) and also show that they can be used for cross-amplification in 4 other Taxus species (T. cuspidata Sieb. et Zucc., T. fuana Nan Li et R. R. Mill., Amentotaxus argotaenia (Hance) Pilger, and Pseudotaxus chienii (Cheng) Cheng).

\section{MATERIAL AND METHODS}

Leaves were collected from 77 T. wallichiana var. wallichiana individuals from 3 populations in Yunnan (100.785E, 24.474N), Hengduan Mountain Range (101.693E, 26.742N) and Himalayan Mountain Range $(96.715 \mathrm{E}, 28.826 \mathrm{~N})$. Voucher specimens from the sampled populations were deposited in the herbarium of the Chinese Academy of Forestry (CAF). The leaves were dried using silica gel and stored at $-80^{\circ} \mathrm{C}$ before use in experiments for analyzing SSR primer polymorphisms. Total genomic DNAs were extracted using a DNeasy Plant Kit (QIAGEN) following the manufacturer protocol.

Genomic DNA $(5 \mu \mathrm{g})$ was sequenced on a 454 Life Sciences GS-FLX (Roche) at the Meiji Biotechnology Company (Shanghai, China). The raw sequences were assembled into contigs using Newbler 2.6 software. The sample occupied $12.5 \%$ of a plate and produced 208,698 reads, with an average fragment size of $555 \mathrm{bp} ; 5.8 \%$ of the sequences contained 
microsatellites. All contigs or singletons longer than $100 \mathrm{bp}$ were screened for microsatellites using MSATCOMMANDER version 0.8.2 (Faircloth, 2008) with default parameters (minimumrepeats were $10,6,5,5,5$, and 4 for mono-, di-, tri-, tetra-, penta-, and hexa-nucleotide, respectively). Primers were designed with the software PRIMER3 (Rozen and Skaletsky, 1999). Forward primers were synthesized with a 19 bp M13 tail (5'-CACGACGTTGTAAAACGAC-3') at the 5'-end to allow labeling with a tailed fluorescent dye. All sequences were submitted to GenBank (accession Nos. KP702222 to KP702230).

We evaluated polymorphisms at each locus from the 77 sampled individuals. Although PCR conditions were optimized for each pair of primers, overall, they were similar. Each PCR amplification was performed in a $25 \mu \mathrm{L}$ reaction solution containing approximately $50 \mathrm{ng}$ template DNA, $12.5 \mu \mathrm{L} 2 \mathrm{X}$ mix (Genestar, Beijing, China), 5 pmol each primer, $10.5 \mu \mathrm{L}$ ddH2O. The following amplification program was used: initial denaturation at $94^{\circ} \mathrm{C}$ for $5 \mathrm{~min}$, followed by 35 cycles at $94^{\circ} \mathrm{C}$ for $30 \mathrm{~s}, 55^{\circ} \mathrm{C}$ for $30 \mathrm{~s}$, and $72^{\circ} \mathrm{C}$ for $45 \mathrm{~s}$ extension; with a final extension step at $72^{\circ} \mathrm{C}$ for $5 \mathrm{~min}$. The PCR products were further monitored and genotyped using a capillary electrophoresis system (CEQ 8000 Genetic Analyzer, Beckman Coulter). The results were exported to the Genomelab Genetic Analysis System.

The microsatellite loci were assayed in 4 Taxaceae species, namely, T. cuspidata Sieb. et Zucc., T. fuana Nan Li et R. R. Mill., A. argotaenia (Hance) Pilger, and P. chienii (Cheng) Cheng), and in 2 varieties of $T$. wallichiana, namely mairei and chinensis. PCR was performed as described above. The products were visualized on a $1.5 \%$ agarose gel run at $110 \mathrm{~V}$ for 20 min along with DL2000 Marker (Takara Biotechnology, Beijing, China); the gel was stained with GoldenView and photographed using the Bio-Rad Gel Documentation System. The loci were considered successfully amplified when at least one band of the expected size was observed.

The number of alleles, size range, expected heterozygosity $\left(H_{E}\right)$ and observed heterozygosity $\left(H_{\mathrm{O}}\right)$ were quantified using POPGENE32 software (Yeh and Boyle, 1997). Deviations from Hardy-Weinberg equilibrium (HWE) for each locus and linkage disequilibrium among all loci were assessed by GENEPOP version 4.2 software (Raymond and Rousset, 1995).

\section{RESULTS}

Of the total of 88 primer pairs designed, 48 pairs successfully amplified the expected product but only 9 loci showed polymorphisms. The PCR conditions and the characteristics of these 9 loci are given in Table 1. The allele number $\left(N_{A}\right)$ of these polymorphic loci ranged from 2 (T10) to 20 (T2) (mean 8.44). $H_{\mathrm{O}}$ and $H_{\mathrm{E}}$ varied from 0.0260 to 0.5325 (mean 0.2468 ) and 0.3603 to 0.9231 (mean 0.6987) (Table 1), respectively. Among the 9 new SSR makers, 8 loci in plants from the Yunnan Plateau region, 7 loci in plants from the Hengduan Mountain region, and 8 loci in plants from the Himalayan region showed significant deviation from HWE after Bonferroni correction $(\mathrm{P}<$ 0.05), most likely due to excess homozygosity, or population structure within the samples, or limited sample size and sampling strategy. No significant linkage disequilibrium was detected between any pairs of loci.

Cross-amplification tests were performed for the two other varieties of $T$. wallichiana and four Taxaceae species (Table 2). The nine loci were successfully amplified in the varieties $T$. wallichiana var. mairei and T. wallichiana var. chinensis and species T. fauna and T. cuspidata; two were successful in P. chienii, and one in A. argotaenia. 
Table 2. Cross-species amplification of other species and varieties in Taxaceae.

\begin{tabular}{lccccccccc}
\hline & \multicolumn{10}{c}{ Locus } & \multicolumn{10}{c}{} \\
\hline Species/Variety & T2 & T10 & T20 & T38 & T40 & T41 & T42 & T78 & T85 \\
Taxus wallichiana var. mairei & + & + & + & + & + & + & + & + & + \\
Taxus wallichiana var. chinensis & + & + & + & + & + & + & + & + \\
Taxus fuana & + & + & + & + & + & + & + & + \\
Taxus cuspidata & + & + & + & + & + & + & + & + \\
Pseudotaxus chienii & - & - & \pm & \pm & \pm & - & - & + \\
Amentotaxus argotaenia & - & - & - & \pm & \pm & - & - & \pm \\
\hline
\end{tabular}

Two individuals of each species were screened. $(+)=$ expected size band amplification; $( \pm)=$ unexpected size band amplification; (-) = no amplification.

\section{DISCUSSION}

All novel markers in the present study showed high levels of polymorphism, indicating that these markers will of value to ongoing research into the population genetic structures, population structures, patterns of gene flow, and mating systems of all the species of the genus Taxus in China.

\section{Conflicts of interest}

The authors declare no conflict of interest.

\section{ACKNOWLEDGMENTS}

Research supported by project \#2130211 of the Rescue and Breeding of Rare and Endangered Wild Plants (Taxus). We thank the Key Laboratory of Tree Breeding and Cultivation of State Forestry Administration for help with the experiment. We also thank Shaoshuai Yu of the Chinese Academy of Forestry for help in sample collection.

\section{REFERENCES}

Dubreuil M, Sebastiani F, Mayol M, González-Martínez SC, et al. (2008). Isolation and characterization of polymorphic nuclear microsatellite loci in Taxus baccata L. Conserv. Genet. 9: 1665-1668.

Ellegren H (2004). Microsatellites: simple sequences with complex evolution. Nat. Rev. Genet. 5: 435-445.

Faircloth BC (2008). MSATCOMMANDER: detection of microsatellite repeat arrays and automated, locus-specific primer design. Mol. Ecol. Resour. 8: 92-94.

Farjon A (2001). World checklist and bibliography of conifers. Royal Botanic Gardens, Kew.

Fu LG, Li N and Mill RR (1999). Taxaceae. In: Flora of China 4 (Wu ZY and Raven PH, eds.). Science Press Beijing \& Missouri Botanical Garden Press, St Louis, Missouri, 89-96.

Huang CC, Chiang TY and Hsu TW (2008). Isolation and characterization of microsatellite loci in Taxus sumatrana (Taxaceae) using PCR-based isolation of microsatellite arrays (PIMA). Conserv. Genet. 9: 471-473.

Kalia RK, Rai MK, Kalia S, Singh R, et al. (2011). Microsatellite markers: an overview of the recent progress in plants. Euphytica 177: 309-334.

Li N and Fu LG (1997). Notes on gymnosperms I. Taxonomic treatments of some Chinese conifers. Novon 7: 261-264.

Miao YC, Lang XD, Zhang ZZ and Su JR (2014). Phylogeography and genetic effects of habitat fragmentation on endangered Taxus yunnanensis in southwest China as revealed by microsatellite data. Plant Biol. 16: 365-374.

Raymond M and Rousset F (1995). GENEPOP (version 1.2): population genetics software for exact tests and ecumenicism. J. Hered. 86: 248-249.

Rozen S and Skaletsky H (1999). Primer3 on the WWW for general users and for biologist programmers. Methods Mol. Biol. 132: 365-386. 
Xinqiang H, Jinxin L, Yushi H, Xianpu W, et al. (1996). Comparison among threatened categories of conifers from China. Chin. Biodiv. 4: 45-51.

Yang JB, Li HT, Li DZ, Liu J, et al. (2009). Isolation and characterization of microsatellite markers in the endangered species Taxus wallichiana using the FIASCO method. HortScience 44: 2043-2045.

Yeh F and Boyle TJ (1997). Population genetic analysis of codominant and dominant markers and quantitative traits. Belg. J. Bot. 129: 157.

Zalapa JE, Cuevas H, Zhu H, Steffan S, et al. (2012). Using next-generation sequencing approaches to isolate simple sequence repeat (SSR) loci in the plant sciences. Am. J. Bot. 99: 193-208.

Zhou Y, Chen G, Su Y and Wang Z (2009). Microsatellite loci from Taxus chinensis var. mairei (Taxaceae), an endangered and economically important tree species in China. Front. Biol. China 4: 214-216. 